\title{
Extending learning objects by means of social networking
}

\author{
Julià Minguillón ${ }^{1}$, M. Elena Rodríguez ${ }^{1}$, and Jordi Conesa ${ }^{1}$ \\ Universitat Oberta de Catalunya, Spain \\ \{jminguillona, mrodriguezgo, jconesac\}@uoc.edu
}

\begin{abstract}
Learning objects have been the promise of providing people with high quality learning resources. Initiatives such as MIT OpenCourseWare, MERLOT and others have shown the real possibilities of creating and sharing knowledge through Internet. Thousands of educational resources are available through learning object repositories. We indeed live in an age of content abundance, and content can be considered as infrastructure for building adaptive and personalized learning paths, promoting both formal and informal learning. Nevertheless, although most educational institutions are adopting a more open approach, publishing huge amounts of educational resources, the reality is that these resources are barely used in other educational contexts. This paradox can be partly explained by the difficulties in adapting such resources with respect to language, e-learning standards and specifications and, finally, granularity. Furthermore, if we want our learners to use and take advantage of learning object repositories, we need to provide them with additional services than just browsing and searching for resources. Social networks can be a first step towards creating an open social community of learning around a topic or a subject. In this paper we discuss and analyze the process of using a learning object repository and building a social network on the top of it, with respect to the information architecture needed to capture and store the interaction between learners and resources in form of learning object metadata.
\end{abstract}

Keywords: Learning Objects, Repositories, Social Networks, Metadata

\section{Introduction}

Virtual learning environments (VLEs) are nowadays in widespread use. From pure virtual universities to traditional ones that offer learners a blended learning model, the use of web-based tools for teaching and learning has been steadily increasing, as described in [17]. From an institutional point of view, VLEs may serve also as a mechanism for content management and dissemination. In fact, most universities have a digital library which reproduces the services from a traditional one, plus adding additional features. But learning resources are not limited to books and traditional paper based publications. Learning in a VLE involves the use of a wide variety of learning objects, including examples and 
exercises, simulations, multimedia documents, etc. These elements must be not only stored but also fully integrated into the learning process, helping learners to better contextualize these small chunks of knowledge. Browsing and searching for these resources should be a truly learning experience. Therefore, learning object repositories should be designed taken into account not only the institutional requirements (preservation and dissemination), but also the needs of the final users, teachers and especially learners, as well as the specific particularities of learning objects.

The concept of a learning object has been deeply discussed many times in the literature since its appearance and massive adoption [19]. Many authors have provided their own definition, which has not helped to clearly convey the concept, causing confusion and constant reformulations [11]. Nevertheless, most of the existing definitions have three main characteristics in common: learning objects are available in digital format; they are described using metadata according to proposed standards formats, and they are oriented to maximize reusability by breaking the resources into pieces that can be reused independently.

On the other hand, institutional repositories are becoming a basic piece of the infrastructure of any educational institution [9]). According to [7], repositories are differentiated from other digital collections because the content is deposited in the repository together with its metadata; such content is also accessible through a basic set of services (i.e. put, get, search, etc.). Depending on the specific needs of the community using the repository, this will provide additional tailored services, but all repositories should at least provide two basic ones: content preservation and content reusing [1]. In particular, learning object repositories (as a specific case of institutional repositories) become a key element for supporting a user centered learning process, combining the services offered by digital libraries with the flexibility of directly providing contents through a simple interface [3]. Nevertheless, the most important requirement to ensure a successful repository states that it should originate from the genuine need of a community [12]. Therefore, any institutional repository will be only half successful if it does not attract, generate and support a community of learners. In this sense, small thematic repositories seem to be a good option. Creating a learning object repository is not a simple task but it should be accomplished from a bottom-up approach (i.e. a group of teachers) with a minimum institutional support (mostly from the IT support office), although there are several preliminary questions that must be addressed [10] with respect to users' perspective. We propose an information architecture embedded into learning object metadata in order to capture all the interactions between learners and learning objects.

This paper is organized as follows: Section 2 describes a learning object repository on Statistics which is used to exemplify the need of building a social network on top of it. Section 3 describes a layer of services based on social networking for improving interaction between learners and learning objects. Section 4 defines the information architecture needed to support the interactions between learners and learning objects. Finally, Section 5 summarizes the main conclusions drawn in this paper and outlines current and future research lines in this topic. 


\section{Case of study: repository on Statistics}

The Universitat Oberta de Catalunya (UOC, in English known as Open University of Catalonia) is an institution which has emerged from the knowledge society. The mission is to provide people with training throughout their lives. UOC's principal aim is to ensure that each student satisfies his/her learning needs in a virtual environment, gaining the maximum benefit from their own efforts. To this end, it offers intensive use of information and communications technologies (ICT), thereby enabling us to overcome the barriers imposed by time and space for offering an educational model based on personalized attention for each individual student. Within the UOC Virtual Campus, each subject has a virtual classroom for teaching and learning process and they are the virtual meeting point for learning activities, following a student centered model [15].

Every semester, more than 4000 students from 11 different degrees enroll into several courses (up to 16) which are related to Statistics. In order to organize this large collection of educational resources (as part of a pilot experience), an open learning object repository with resources on Statistics has been built using DSpace ${ }^{1}$ as platform. DSpace was designed in 2002 by MIT Libraries in collaboration with the Hewlett-Packard Company. Its focus on the submission, storage, access and preservation of research material in digital format makes it well suited to the needs of an institutional or thematic repository, ranging from a few hundred to hundreds of thousands of items, namely learning objects. This repository, named OER, has been designed with the aim of providing learners with a comprehensive vision of the whole knowledge domain of Statistics, trying to make of browsing and searching a true learning experience [6]. In order to do so, learning objects in the repository have been tagged according to the following minimum criteria: every resource is an element part of several taxonomies (one for describing the Statistics domain of knowledge, another for describing the kind of resource and a third one for identifying the course or degree the resource was created for), and it is described by one or more keywords. No other metadata fields are required. Therefore, according to their nature, learning objects may have or not title, author, creation date, etc., so they cannot be accessed by classical retrieval mechanisms used in digital libraries or repositories. In fact, DSpace had to be customized to change the basic fields used for searching and browsing, as well as the workflows related to the process of adding new resources to the repository. The only exception is "title", which is mandatory for DSpace in order to access a given element in the repository.

\section{$3 \quad$ Adding social networking functionalities}

But once the learning object repository is online, it needs to become the center of a community of learners. Indeed, the success of many Web 2.0 communities such as YouTube or flickr lies in that they were able to attract a critical mass of users that either provide contents or add value to the existing contents in

\footnotetext{
${ }^{1}$ http://www.dspace.org/
} 
the site by commenting, rating and bookmarking. If creating a learning object repository from scratch is not possible, another possibility is using an existing one, taking advantage of its reputation, critical mass and other relevant factors [12]. In this sense MERLOT ${ }^{2}$ can be mentioned as an example of a LOR that has succeeded in attracting an active user community, and nowadays MERLOT offers the possibility to navigate the resources through the profiles of registered users. MERLOT has several mechanisms to award recognition to active users that provide high-quality contributions. This helps to create a community around the repository. Nevertheless, in this paper we use the learning object repository described in the previous section as the basis of our proposal.

New learning theories such as connectivism [16] establish that learning is produced during the process of establishing new relationships between contents and concepts, rather than in the already acquired knowledge. Learning object repositories are important elements in the network built by the learner during his or her learning process, as they store not only the learning resources but also all the details of the learning experience itself (with respect to the learner). Learning occurs anytime, anywhere, learners do not need to go to a specific place to have a learning experience, on the contrary, they should be able to learn whatever, whenever, wherever. In this sense, social networks provide a basic support for this practice, but not contents. Learners do not need to know everything; it is the ability to create, analyze and share connections between resources that generates knowledge. In this sense, learning is more than just content; this is just the infrastructure for the learning process [18]. Therefore, in order to promote the reuse of open educational resources, we propose to bridge both worlds. From the one side, by means of institutional repositories built on a top-down approach, more aimed towards preservation rather than promoting reuse and, from the other side, communities of practice and learning in the shape of social networks. We can combine the best of both worlds: reliable and permanent handles for welldescribed resources in learning object repositories (where the contents are) with dynamic services available through social networks and web 2.0 tools (where the learners are). So, once a learner finds (and uses) a learning resource, whatever the source is, he or she should be able to perform the following actions on it:

- Comment: in order to promote a continuous improvement of resources, learners should be able to make comments, place questions, correct small mistakes and so, using communication spaces directly related to the learning object, not separately. These annotations can create a micro-community of learning around a given learning object.

- Rate: using a Likert-type scale, stars (from 0 to 5 or 10) or any other mechanism, the learner should be able to express his or her valuation of the resource. This information can be used to rank learning objects according to their explicit popularity.

- Favorite: for those resources that really capture learners interest, it should be possible to mark them as a very valuable resource, analogously to what users do with links.

\footnotetext{
${ }^{2}$ http://www.merlot.org/merlot/index.htm
} 
- Tag: learners should be able to describe learning resources using their own keywords, as a way of self-organizing concepts through the use of small notes.

- Share: all of the previous actions should be shared using learners usual communication channels, such as twitter, facebook, delicious and so.

- Subscription: finally, learners should be aware of all interactions occurring around a specific resource, being able to subscribe to a given learning object, using RSS or any other similar technology.

Therefore, for each resource there is a list of triplets \{user, action, value\} which defines all the interactions generated around such resource. Each triplet has also a timestamp associated with it. These interactions can be stored separately, although we propose to manage them as information related to each learning object, by including them as additional metadata, extending the description of such learning object. On the other hand, from an institutional perspective, all these interactions between learners and resources can be stored and analyzed in order to provide a better understand of the learning process [5], as well as providing useful information to the searching engine, improving searching and browsing results. Analyzing comments may also help to detect problems with certain resources and correct them. Finally, analyzing tags can be also a useful source of information for improving metadata describing educational resources.

In order to implement the services aforementioned, it is important to separate the storing capabilities of the learning object repository from those more related to searching and browsing, adding to them the services that will provide learners with a better personalized management of learning resources. This separation can be implemented through the use of an API (Application Programming Interface) which services can be accessed through a more user friendly module. One possibility is using a content management system such as Drupal ${ }^{3}$, for example, as described in [4], although implementation issues are out of the scope of this paper. In the next section we will discuss how to capture and store all these knowledge sharing as metadata within each learning object.

\section{Information architecture}

Hereby we present the information architecture that allows to support the services presented in the previous section. Firstly, and from a conceptual point of view, we identify the classes of interest, their properties as well as the relevant relationships among theses classes and the integrity constraints that must be enforced. Classes, properties and relationships are depicted in figure 1 by means of an UML class diagram. Afterwards we discuss how to integrate all these elements in a metadata schema based on the IEEE LOM standard.

\subsection{Modeling learner annotations on learning objects}

Every available service can been seen as a kind of annotation (see Figure 1) that collects data provided by one learner about one learning object at a given point

\footnotetext{
${ }^{3}$ http://drupal.org/
} 
of time. The specialization of annotations is left as incomplete; this implies that other kinds of annotations could be added in order to fulfill new requirements. Depending on the kind of annotation under consideration, the data provided by the learner with his or her action can differ. In the case of tags, for instance, they are represented as a list of keywords. Each learner can only declare one list of keywords for a given learning object. In case the learner wants to add a new keyword to a previously tagged learning object, the keyword will be simply added to such list. On the other hand, rates can be modeled by pairs representing scale (we assume that the use of different scales is allowed) and valuation properties. For a given scale, each learner can only provide a valuation for a concrete learning object. Any change performed by the learner on a given valuation will be interpreted as a valuation update replacing the previous one. Scales can impose additional constraints, for example restrictions on the allowed range of values. At its own, comments are captured by means of a description which represents the content itself associated to the comment. Learners can state an unlimited number of comments on every learning object. Given that the date (which can be seen as a timestamp that could be automatically generated by the system) is recorded for any annotation, the set of comments produced by each learner can be assessed from a temporal (or historical) perspective. When the annotation deals with the sharing of a learning object, it is required to characterize the communication channel used by the learner. Each learner can share a concrete learning object as many times as he or she wants to, although the fact that a learning object has been shared through a specific channel is stored only once. Finally, subscription and favorite do not require to add additional properties, although they have been made explicit for the sake of semantic expressiveness. Every learner, for a given learning object, only needs to subscribe and mark it as favorite once.

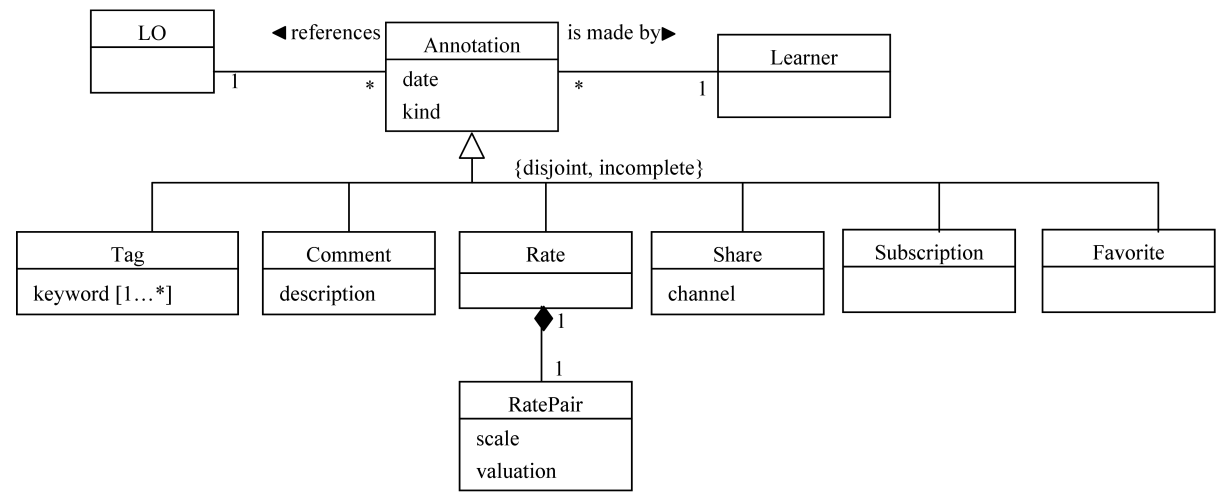

Fig. 1. Conceptual information architecture. 


\subsection{Integration in the LOM based metadata schema}

For implementation purposes, the conceptual information architecture aforementioned must be integrated inside the OER repository. Amongst others, this implies to make decisions about how all required data must be structured. In the e-learning field, several standards and specifications have been proposed in the form of metadata schemas in order to capture both user and learning objects properties. Given that our main objectives are to promote the reuse of learning objects and their continuous improvement based on learners' annotations, we consider that such annotations must be seen as an essential part of the description of the learning object itself. Therefore, we need to develop a metadata schema for learning objects that fulfills our data requirements. Ideally, the developed metadata schema should be compliant with some standard metadata schema for learning objects, in order to ensure interoperability within different repositories of the same institution or with similar repositories developed by other institutions. The most popular metadata schema for describing learning objects are those proposed by Dublin Core and LOM. For our purposes, Dublin Core metadata schema does not provide any support. In the case of LOM we find partial support through the Annotation category. Currently now, the learning object repository based on DSpace is using Dublin Core. Nevertheless, it is possible to map items between the two metadata schemes, so they are fully interoperable. Furthermore, DSpace provides also mechanisms to incorporate different metadata schemes for describing items in the repository.

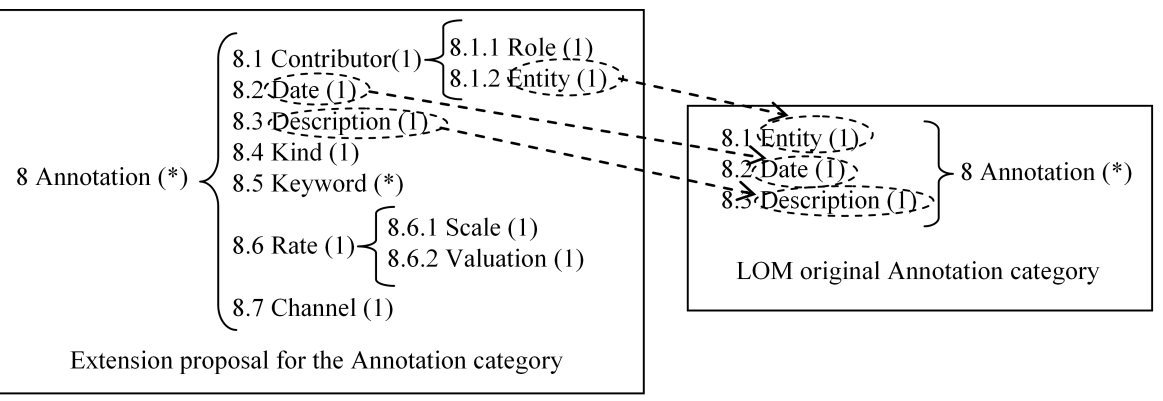

Fig. 2. Proposed extension for the Annotation category.

According to LOM, the Annotation category provides comments on the educational use of the learning object that is being described, as well as information on when and whom the comments were created. LOM also specifies that comments are provided by educators or educational institutions. The metadata elements included in this category are Entity (which represents the people or organization that created the annotation), Date (that collects when the annotation was created) and Description (which captures the content of the annotation). Obviously, LOM allows that the metadata schema can be extended to 
accomplish the particular requirements of any organization, although in order to maximize interoperability, extended data elements should not replace data elements in the LOM structure. The metadata elements that we propose for the Annotation category include not only the LOM original metadata elements, but also new ones. Our proposal is graphically depicted in Figure 2 following the common LOM style, i.e. metadata elements are numbered according to the category they belong and the number of values allowed for each metadata element is shown in parenthesis.

In order to distinguish between educator and learner annotations, we propose an aggregated metadata element, named Contributor, that collects pairs $\{$ role, entity\}. The role element represents the kind of user \{educator, learner $\}$ that creates the annotation. The values to be assigned to this element can be organized as a list of appropriated values (vocabulary, in terms of LOM). On the other hand, the entity element corresponds to the original LOM metadata element entity. This correspondence is shown in Figure 2 by means of a dashed arrow. In a similar way, the date and description properties identified in our conceptual information architecture can be directly mapped (see again figure 2) to the original LOM metadata elements date and description. While date metadata is applicable to any annotation irrespective of its kind, the description metadata is only applicable to annotations of kind comment. In other words, that means that a description is an specific property of comments and it does not have sense to apply it to any other kind of annotation (for example, tags, rate or share annotations). The same reasoning can be applied to metadata elements keyword, rate pair and channel that are only applicable, respectively, to tag, rate and share annotations. Therefore, our API must control that learners annotate learning objects in the appropriate way and according to the desired behavior.

The specific type of annotation is represented by means of the metadata kind. In the case of comments, their kind could be further refined (question, mistake detection, review, suggestions for use etc.) as it has been explained in section 3. Although the values associated to this metadata could be expressed as a vocabulary, the idea of organizing metadata values in the form of a vocabulary is quite problematic when the number of values for the vocabulary increases. As different empirical analysis reveal ([2], [8]), the use of vocabularies does not lead to consistent descriptions. Differences occur, given that the description partially depends on persons. Therefore the value assigned to a metadata is based on the interpretation that people do about the available values. For this reason we suggest to express the values associated to the metadata element kind in a taxonomic way. In [14] the reader will find a complete discussion about how to develop taxonomies of interest in the learning objects realm from a common sense ontology.

Finally, and although our proposal apparently could compromise interoperability, it is important to note that this can be solved in case we define a procedure that allows to export our metadata records according to the metadata schema proposed by LOM. In our case, this can be easily done by transferring 
the values of the added metadata to the original LOM metadata, more specifically to the original LOM metadata element description. For example, in the case of an annotation of kind rate, the original LOM metadata description will include that a learner has made a valuation of the learning object according to an specific scale. Summing up, the procedure transforms semantics that our model considers in an explicit way in implicit semantics. The disadvantage here is the implicit semantics only is human understandable.

\section{Conclusions}

Learning object repositories are nowadays a basic piece of any virtual learning environment, but learners still need to go to the repository in order to find learning resources. Furthermore, once a given resource is found, learners are only left with the possibility of consuming it, but nothing else. In order to integrate the resource into the learners learning process, a collection of basic services should be built on top on it, with the aim of creating a true community (even at a small scale) around such resource, making of it a valuable asset.

In this paper we have described a set of services that can be implemented on top of any learning object repository, with the aim of promoting the use of learning objects among learners, allowing them to contextualize learning objects into their own learning process. The interaction between learners and learning objects can be captured and stored in form of metadata into every learning object, allowing us to analyze it in order to extract useful information for improving the learning object repository and users' experience. We have proposed an extension to the IEEE LOM standard that takes into account the nature of the services, adding all the necessary detail but without compromising the standard, thus ensuring interoperability.

Current and future research in this topic should include the design of new user interfaces for searching and browsing learning resources [13] taking into account the information gathered from repository usage. Analyzing interaction throughout long periods of time (i.e. an academic semester) will reveal also interesting facts about the quality of learning objects in terms of access and usage, improving overall system design. Finally, the use of semantic web technologies for providing personalized services is also an interesting issue, including both accessibility and mobility aspects, thus promoting a true open access to learning resources anytime, anywhere.

\section{Acknowledgements}

This paper has been partially supported by Spanish projects TIN2010-21715-C02 and TIN2008-00444.

\section{References}

1. Akeroyd, J.: Information management and e-learning. some perspectives. Aslib Proceedings: New Information Perspectives 57(2), 157-167 (2005) 
2. Cechinel, C., Sánchez-Alonso, S., Sicilia, M.A.: Empirical analysis of errors on human-generated learning objects metadata. In: Sartori, F., Sicilia, M.A., Manouselis, N. (eds.) Metadata and Semantic Research. Third International Conference. Communications in Computer and Information Science, vol. 46, pp. 60-70. Springer-Verlag (September-October 2009)

3. Conway, P.: Modeling the digital content landscape in universities. Library Hi Tech 26(3), 342-354 (2008)

4. Coombs, K.: Drupal done right. Library Journal 134(19), 30-32 (2009)

5. Ferran, N., Casadesús, J., Krakowska, M., Minguillón, J.: Enriching e-learning metadata through digital library usage analysis. The Electronic Library 25(2), 148-165 (2007)

6. Ferran, N., Guerrero-Roldán, A.E., Mor, E., Minguillón, J.: User centered design of a learning object repository. In: Kurosu, M. (ed.) Proceedings of the 1st International Conference on Human Centered Design, HCI International. Lecture Notes in Computer Science, vol. 5619, pp. 679-688. San Diego, CA (July 2009)

7. Heery, R., Anderson, S.: Digital repositories review. Tech. rep., JISC (2005)

8. Kabel, S., de Hoog, R., Wielinga, B., Anjewierden, A.: Learning objects: Vocabularies and empirical investigation of consistency. Journal of Educational Multimedia and Hypermedia 13(4), 405-425 (2004)

9. Lynch, C.A.: Institutional repositories: Essential infrastructure for scholarship in the digital age. ARL (226), 1-7 (February 2003)

10. Margaryan, A., Littlejohn, A.: Repositories and communities at cross-purposes: issues in sharing and reuse of digital learning resources. Journal of Computer Assisted Learning 24(4), 333-347 (2009)

11. McGreal, R.: Learning objects: a practical definition. International Journal of Instructional Technology and Distance Learning 1(9) (September 2004)

12. McNaught, C.: Are learning repositories likely to become mainstream in education? In: Proceedings of the 2nd International Conference on Web Information Systems and Technologies. pp. IS9-IS17. Setubal, Portugal (Apr 2006)

13. Minguillón, J.: Learner-centered learning object repositories: personalization and interaction issues. In: 8th European Conference on e-Learning. p. Keynote speaker. Bari, Italy (October 2009)

14. Rodríguez, M.E., Conesa, J., Sicilia, M.A.: Clarifying the semantics of relationshis between learning objectsa. In: Sartori, F., Sicilia, M.A., Manouselis, N. (eds.) Metadata and Semantic Research. Third International Conference. Communications in Computer and Information Science, vol. 46, pp. 35-47. Springer-Verlag (September-October 2009)

15. Sangrà, A.: A new learning model for the information and knowledge society: The case of the UOC. International Review of Research in Open and Distance Learning 2(2) (Jan 2002)

16. Siemens, G.: Connectivism: A learning theory for the digital age. International Journal of Instructional Technology and Distance Learning 2(1), 3-10 (2005)

17. Taylor, J.C.: Distance education: the fifth generation. In: Proceedings of the 19th ICDE World Conference on Open Learning and Distance Education. Vienna, Austria (1999)

18. Wiley, D.: Peer-to-peer and learning objects: The new potential for collaborative constructivist learning online. In: Proceedings of IEEE International Conference On Advanced Learning Technologies. pp. 494-495 (2001)

19. Wiley, D.: The Instructional Use of Learning Objects. Agency for Instructional Technology (Jan 2002) 